\title{
Computer Simulation of Breast Reduction Surgery
}

\author{
Augusta Cardoso - Gustavo Coelho • \\ Horácio Zenha • Vera Sá • Georgi Smirnov • \\ Horácio Costa
}

Received: 28 February 2012/ Accepted: 17 September 2012

(C) Springer Science+Business Media New York and International Society of Aesthetic Plastic Surgery 2012

\begin{abstract}
Background Plastic surgery of the breast, particularly breast reduction, is considered difficult. It can become a challenge for a less experienced surgeon to understand exactly what to do when facing a particular type of breast and how to avoid unsatisfactory results.

Methods The goal of this study was to create a computer model of the breast that provides a basis for the simulation of breast surgery, particularly breast reduction. The reconstruction of elastic parameters is based on observations of the breast with the patient in different positions.

Results It is shown that several measurements with the patient in different positions allow one to choose the parameters of the model and determine the elastic coefficients of the breast and the skin. The geometry of the breast before and after surgery is simulated. A qualitative study of the incision parameters' influence on the final geometry of the breast is presented.

Conclusion The developed methodology and software allow one to estimate the form of the breast after the surgery by knowing its form before surgery and taking into consideration the parameters of incision applied by the
\end{abstract}

\footnotetext{
A. Cardoso $(\varangle) \cdot$ G. Coelho $\cdot$ H. Zenha $\cdot$ H. Costa

Centro Hospitalar de Vila Nova de Gaia/Espinho EPE - Portugal,

Rua Conceição Fernandes, 4434-502 Vila Nova de Gaia,

Portugal

e-mail: acardoso@chvng.min-saude.pt

V. Sá

Faculdade de Ciências, Universidade do Porto, Rua do Campo

Alegre, 687, 4169-007 Porto, Portugal

G. Smirnov

Centro de Física, Departamento de Matemática e Aplicações, Universidade do Minho, Campus de Gualtar, 4710-057 Braga, Portugal
}

surgeon at the time of surgery. The described approach can be used for the qualitative and quantitative study of breast reduction surgery with a satisfactory result.

Level of Evidence $V$ This journal requires that authors assign a level of evidence to each article. For a full description of these Evidence-Based Medicine ratings, please refer to the Table of Contents or the online Instructions to Authors http://www.springer.com/00266.

Keywords Breast reduction surgery - Surgery planning · Breast modeling $\cdot$ Chassaignac space $\cdot$ Finite element method

\section{Introduction}

Breast reduction is one of the most common procedures in breast surgery and has been gaining in importance, especially in women over 40 years of age [12, 13, 15, 20, 23]. Mastering the techniques takes time and it can become a challenge for a less experienced surgeon to understand exactly what to do when facing a particular type of breast. The aim of this work was to develop a computational model that allows one to predict the final breast geometry according to the incision marking parameters. This model can be used in surgery simulators that provide preoperative planning and training.

Although the number of studies on breast soft tissue modeling has increased significantly during the last few years [7, 17, 24], the development of an adequate breast model continues to be a problem. The main areas where breast modeling is applicable are aesthetic surgery $[2,4,6$, $18,21,22]$ and medical imaging analysis [3].

The breast reduction surgery simulator described in this article is based on a numerical solution of what we call a 
general problem of plastic surgery. From the mathematical point of view, this is a problem of calculus of variations with unusual boundary conditions known as knitting conditions. The breast tissue is considered a hyperelastic material, i.e., a material satisfying the standard thermodynamic axiom of non-negative work in closed processes [11]. Although most soft tissues are incompressible [9], we consider the breast a compressible neo-Hookean material [27]. The complex structure of the breast involves several tissues that form it. Its elastic properties cannot be readily deduced from the elastic properties of the tissues that form it. The compressibility of the breast is an experimental fact. Indeed, breast reduction surgery in which only part of the skin is removed from the breast (breast lift) diminishes the breast volume without removing any part of its internal tissue. The skin has elastic properties that are very different from those of the breast gland and fat tissue, and therefore, must be modeled differently [19]. The breast tissue is modeled using three-dimensional finite elements and the skin is modeled using two-dimensional finite elements [26]. Our computational simulations show that the skin's elasticity properties significantly influence the form of the breast before and after surgery. The skin's function is to contain the jelly-like breast tissue. Its modeling is very important to the understanding and predicting of the results of breast reduction.

A realistic breast model is impossible without the so-called Chassaignac space [5]. This tissue plays a special role in breast mobility and is responsible for the connection between the breast and the chest (Fig. 1). Here we present a breast model involving the Chassaignac space, the latter is modeled as a mass-spring system [16].

There are many studies dedicated to the problem of elastic parameters determination [1, 8]. In this work, the elastic parameters are determined by observing breast geometry. This methodology is new and is based on the fact that when the patient changes her position (upright, prone, on the back, etc.), the breast geometry also changes and these transformations depend on the elastic properties of the breast. The necessary measurements do not require special equipment and can be fulfilled in a usual consulting room. The software developed for breast reduction modeling allows one to predict the final breast geometry according to the incision marking parameters. The comparison of our simulations with a real case gives satisfactory results.

\section{Methods}

In this section we describe the methodology, the necessary measurements, and the software developed to predict the breast form after surgery.

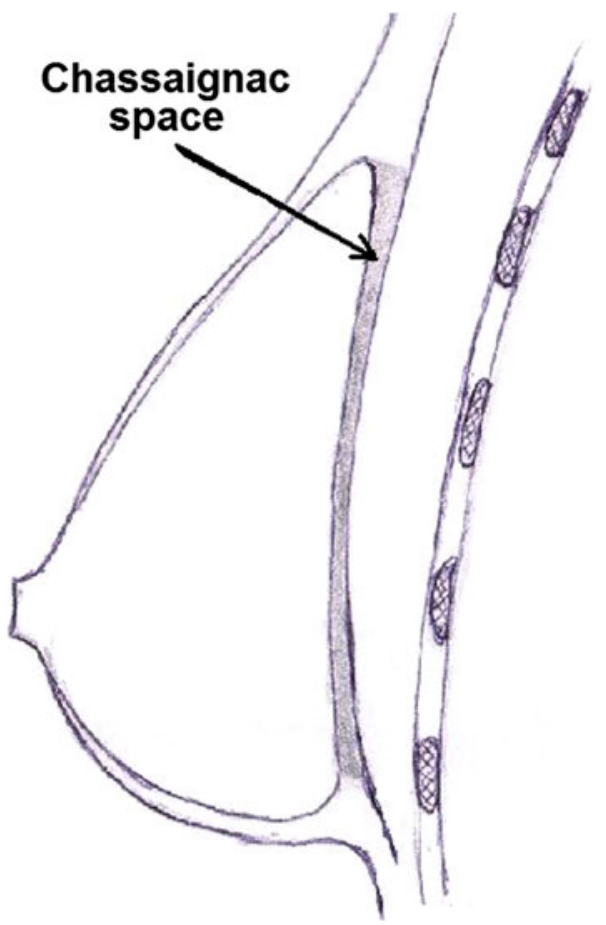

Fig. 1 Transverse section of the breast showing the Chassaignac space

Mathematical Formulation of the Problem

To make the articled self-contained, we include a short description of the mathematical model used in our simulations. This part is directed at specialists in numerical modeling of soft tissues. We give a rigorous formulation of the problems numerically solved by our software to predict the results of plastic surgery. The reader who is not interested in the theoretical basis of our study can skip this section and go directly to the practical part without loss of continuity.

The boundary $\Gamma$ of the elastic body $B$ is represented as $\Gamma=\Gamma_{1} \cup \Gamma_{2} \cup \Gamma_{3} \cup \Gamma_{4}$, where $\Gamma_{3}=\Gamma_{+} \cup \Gamma_{-}$. This means that we consider the boundary of the elastic body as being formed by a fixed part $\Gamma_{1}$, a free part $\Gamma_{2}$, a part $\Gamma_{3}$ where two of the incised tissues are sutured to each other, and another part where the incised tissues are sutured to a fixed surface $\Gamma_{4}$. Let $f$ be the deformation of the body and let $W$ be the strain-energy density [11]. Consider the following variational problem:

$I(f)=\int_{\mathrm{B}} W(\nabla f(p)) d p \rightarrow \inf$

with the boundary conditions:

$$
\begin{aligned}
& f(p)=p, p \in \Gamma_{1}, \\
& f(g(p))=f(p), p \in \Gamma_{+}, \\
& h(f(p))=0, p \in \Gamma_{4},
\end{aligned}
$$


where $g: \Gamma_{+} \rightarrow \Gamma_{-}$is a map. It can be fixed or variable and describes the suturing. We call problem (1)-(4) a general plastic surgery problem.

As we have mentioned before, in the plastic surgery problem we consider a neo-Hookean compressible [27] material. This means that the strain-energy density function is given by

$W(F)=\frac{\mu}{2}\left(\operatorname{tr}\left(F F^{T}\right)-3-2 \ln (\operatorname{det}(F))\right)+\frac{\lambda}{2}(\operatorname{det}(F)-1)^{2}$

where $F=\nabla f$ is the Jacobi matrix of $f$. The parameters $\lambda$ and $\mu$ are different for the skin and the interior tissue.

This problem has already been studied [6, 21, 22]. In previous works the finite element method equations are deduced for this problem and the problem is placed among other elasticity theory problems.

To model the Chassaignac space, we consider a massspring system situated at the breast base. From the mathematical point of view, this means that we add the functional

$\int_{\Gamma_{1}} c(p)(f(p)-p)^{2} d p$

to functional (1). Here, $c(p)$ is a given function.

\section{Computational Aspects}

We use the finite element method [26] to obtain a reliable computational model of the breast. The breast tissue is modeled using three-dimensional finite elements and the skin is modeled using two-dimensional finite elements. The Chassaignac space is modeled by a mass-spring system.

We assume that there is a "neutral state" of the breast, i.e., a state of the breast in which all forces (elastic and mass) are zero. Although the existence of this state is a very strong and unrealistic hypothesis, its assumption is absolutely necessary for the modeling.

The modeling of breast reduction surgery includes the following main steps: (1) determination of the elastic parameters $\lambda$ and $\mu$ in (5) and of the original breast's neutral state, (2) incision of the tissue to be removed, (3) tissue suturing, and (4) determination of the breast's final neutral state. The first step is described in the next section. The second one is a simple geometric construction applied to the breast in the neutral state. To fulfill step 3, the most complicated step, we numerically solve variational problem (1)-(4). Finally, to get the breast's final neutral state we set all stresses equal to zero. This last step can be considered as breast recovery modeling.

To obtain the prediction of surgery for states other than the neutral one, e.g., when the patient is standing up, we have to apply mass forces to the previously obtained neutral state and solve problems (1) and (2).

The Methodology for Determination of the Breast's Elastic Parameters

One of the most important parts of this work is the determination of the breast's elastic parameters based on the study of breast deformation as a function of patient position. The corresponding mathematical problem consists of the minimization of the discrepancy between what is observed and what is modeled. The function $W$ (see (5)) depends on a vector of parameters $y=(\lambda, \mu)$. Therefore, the deformation $f$ also depends on $y$. We calculate a finite number of observable geometric characteristics $G(f(p, y))$ obtained as a result of the deformation $f(p, y)$, e.g., $G=\left(y_{\min }, z_{\min }\right)$ (Fig. 2$)$. Let $\hat{G}$ be the result of the observation. To reconstruct the corresponding vector of parameters, $\hat{y}$, our software solves the minimization problem

$$
\underset{y}{\min }|\hat{G}-G(f(p, y))|^{2}
$$

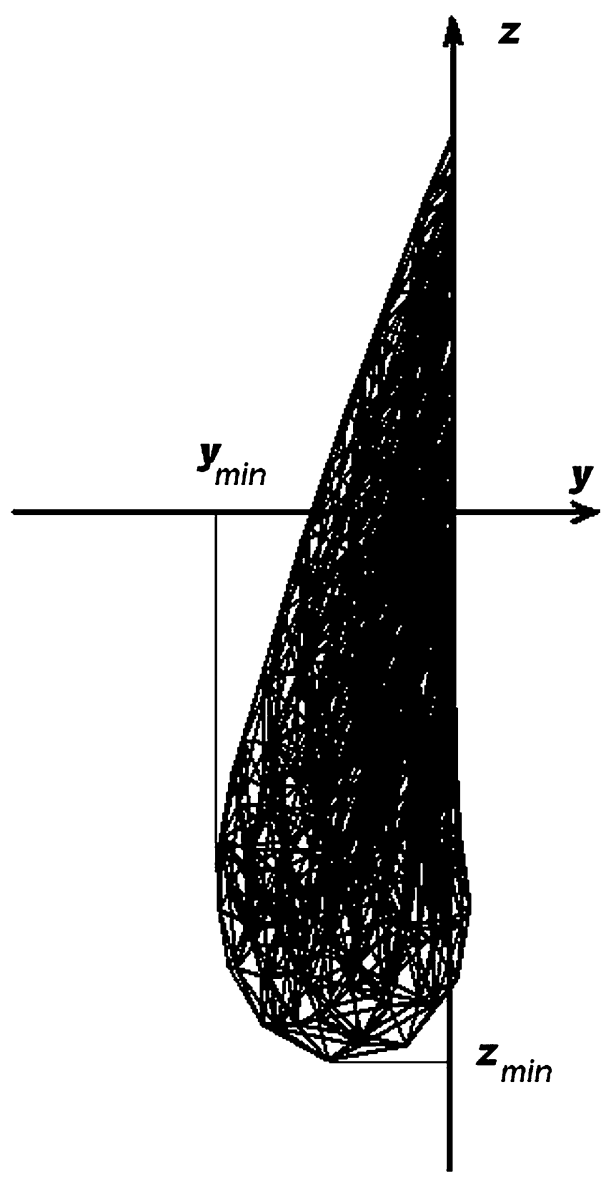

Fig. 2 An example of observable parameters: $y_{\min }$ and $z_{\min }$ 


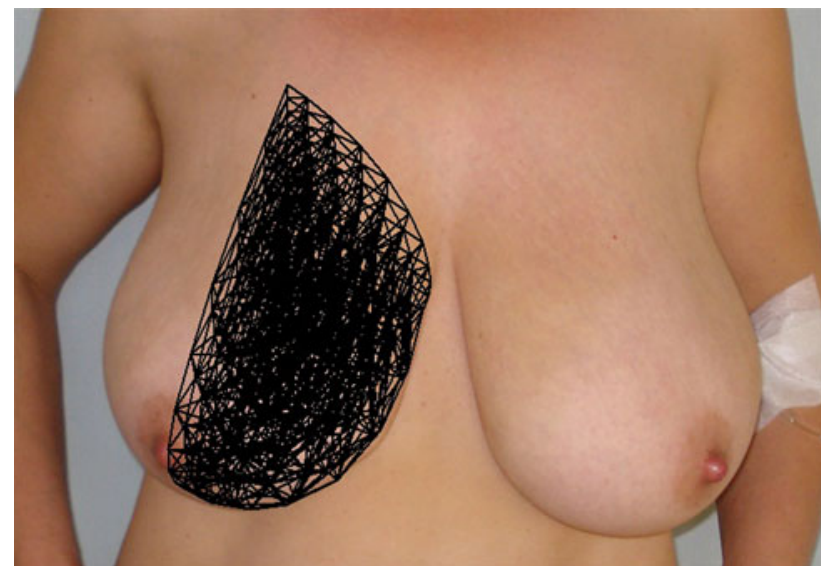

Fig. 3 Preoperative breast model

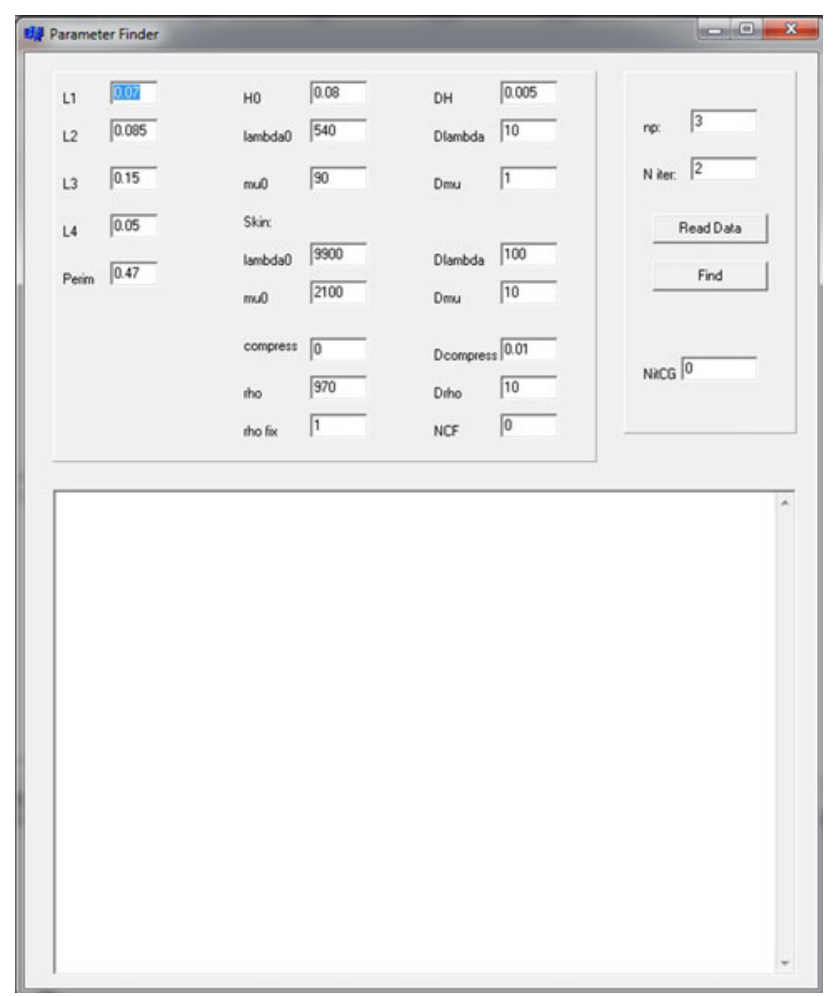

Fig. 4 Screen of the interface of parameter finder

Using this methodology we determine the parameters $\lambda$ and $\mu$ in (5) and obtain the preoperative breast model (Fig. 3).

\section{Software}

The application developed to model breast reduction surgery and used in our simulations runs under the Windows operating system and contains three blocks: Parameter Finder, Breast, and Viewer. The respective interfaces are shown in Figs. 4, 5, 6.
The user enters the data collected from observation of the breast, including perimeter of the base; height; extreme values of $y$ and $z$ when the patient is standing, lying supine, and kneeling on fours; and initial guesses for the elastic parameters of the breast and the skin and for tissue density. The program finds the parameters that provide a good fit between measured and simulated data. The obtained elastic parameters and the density are used as data for the Breast program, modeling a breast reduction surgery. The user can choose the incision parameters $a, s, h$, and $d$, described below. As a result, the program generates files containing computational models of preoperative and postoperative breasts when the patient is in a position defined by the angle between the chest and the vertical. The Viewer allows one to observe the preoperative and postoperative breasts from different angles. Parameter Finder and Breast interfaces have textboxes allowing the user to follow the computation processes.

\section{Results}

Now we describe the main results obtained using our computer simulator.

\section{The Influence of Incisions on Final Breast Geometry}

Based on the incisional marking [25] (Fig. 7), we consider the breast a symmetric body. The plane of symmetry is orthogonal to the chest and passes through the nipple dividing the breast into two equal parts. It is assumed that in the neutral state the breast is a spherical cap with a base radius $R$ and height $H$ (Fig. 8). These parameters are also determined from the observations described above.

Due to the symmetry of the model, we consider only half of the breast to simulate the reduction surgery. Moreover, we do not take into account the type of nipple pedicle. Note that the pedicle type influences only the volume of the tissue removed from one or another part of the breast and it is not important for the modeling considered here. The suspensory ligament is not modeled either because it does not play a relevant role in the shape and geometry of the breast. In fact, in cases of moderate to severe mammary hypertrophy, the suspensory ligament has a limited function due to its great laxity.

The surgical scheme is shown in Fig. 8. The breast is incised by the planes $A B D$ and $B C D$. The point $A$ represents a new position for the nipple. The point $C$ belongs to the breast base and $D$ is a point on the breast surface. The curvilinear triangle $B C D$ is then sutured to the "chest," the plane $0 x z$, and the curvilinear triangle $A B D$ is sutured to the plane $0 y z$, which plays the role of the symmetric part of the breast. 
Fig. 5 Screen of the interface of breast

Fig. 6 Screen of the interface of viewer

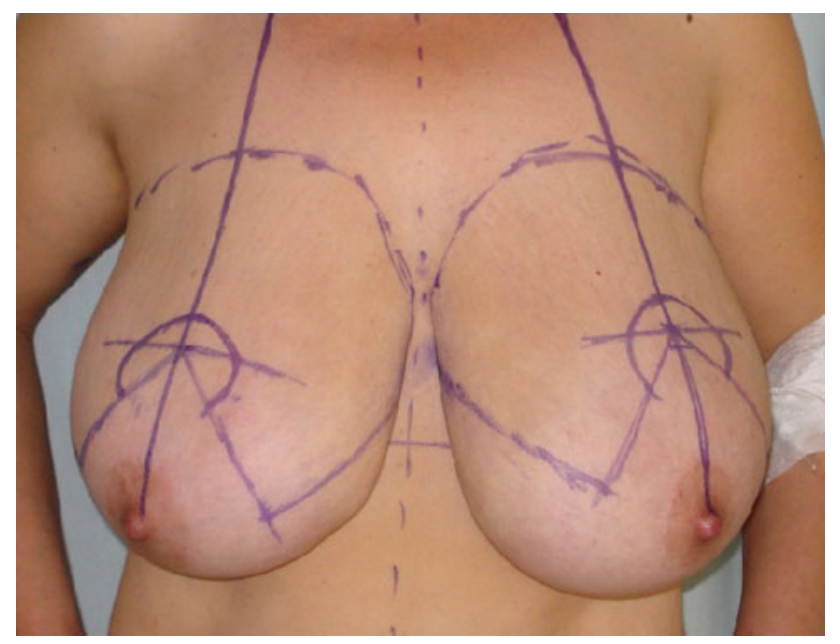

Fig. 7 Cutting lines
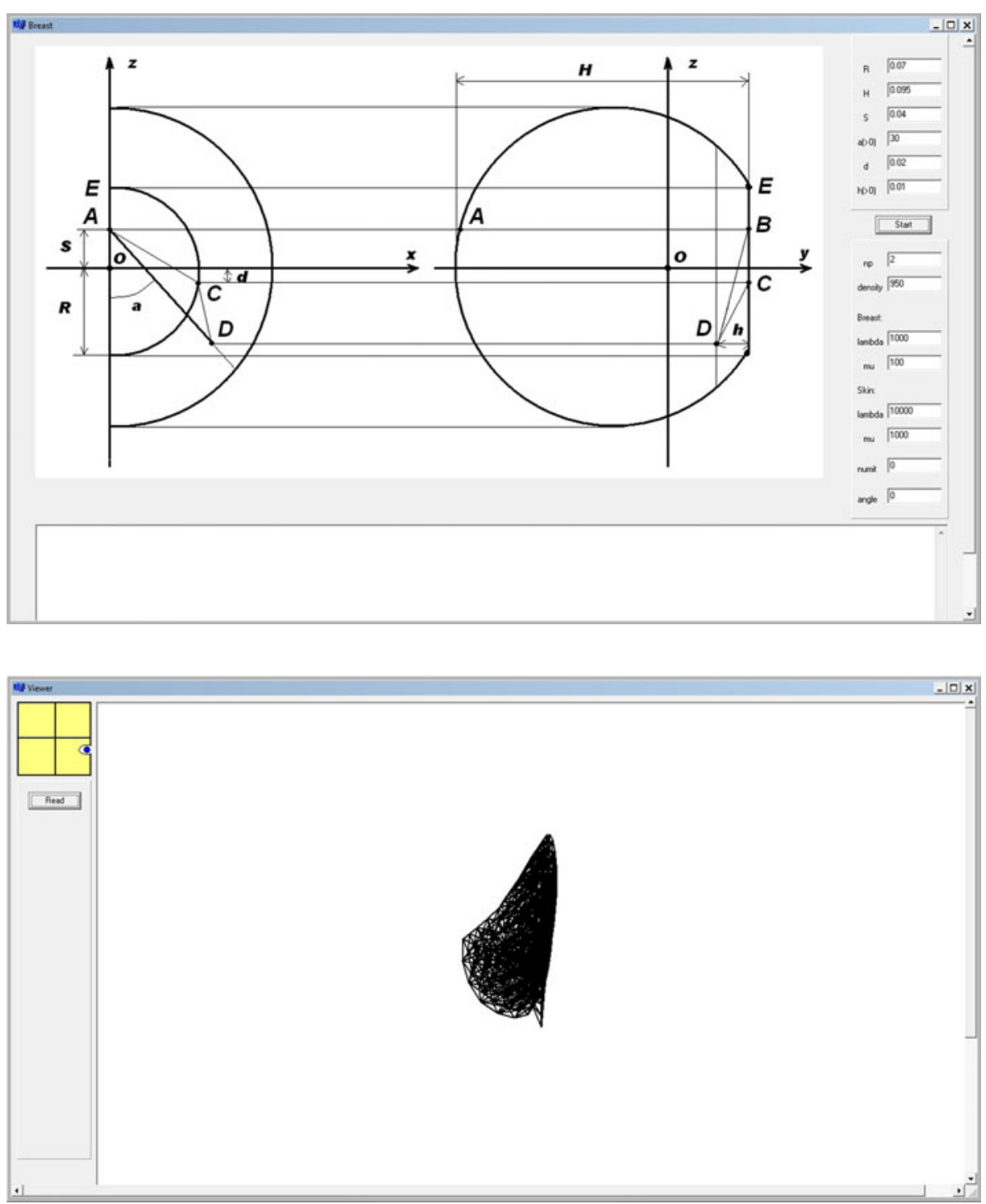

To describe the cutting planes $A B D$ and $B C D$, we use the angle $a$ between the planes $A B D$ and $0 y z$ (Figs. 8 and 9), the distance $s$ between point $A$ and the plane $0 x y$, the distance $d$ between point $C$ and the plane $0 x y$, and the distance $h$ between point $D$ and the base of the breast. It takes several measurements, with the patient in different positions, to choose the parameters of the model and to determine the elastic coefficients of the breast and of the skin according to the methodology described in the previous section.

We have simulated the geometry of breast before surgery (Fig. 3). Then, applying different types of incisions, changing the parameters $h$ and $a$ (Figs. 8, 9), we obtained distinct final geometries (Fig. 10). The result of the surgery is a solution to the variation problem (1)-(4), where $B=$ $B(a, h)$ is the incised breast determined by the parameters $a$ and $h$. A comparison of the computer simulation of breast reduction with real surgery is shown in Fig. 11. 
Fig. 8 Neutral state of the breast and tissue incision scheme depending on the parameters $a, s, h$, and $d$
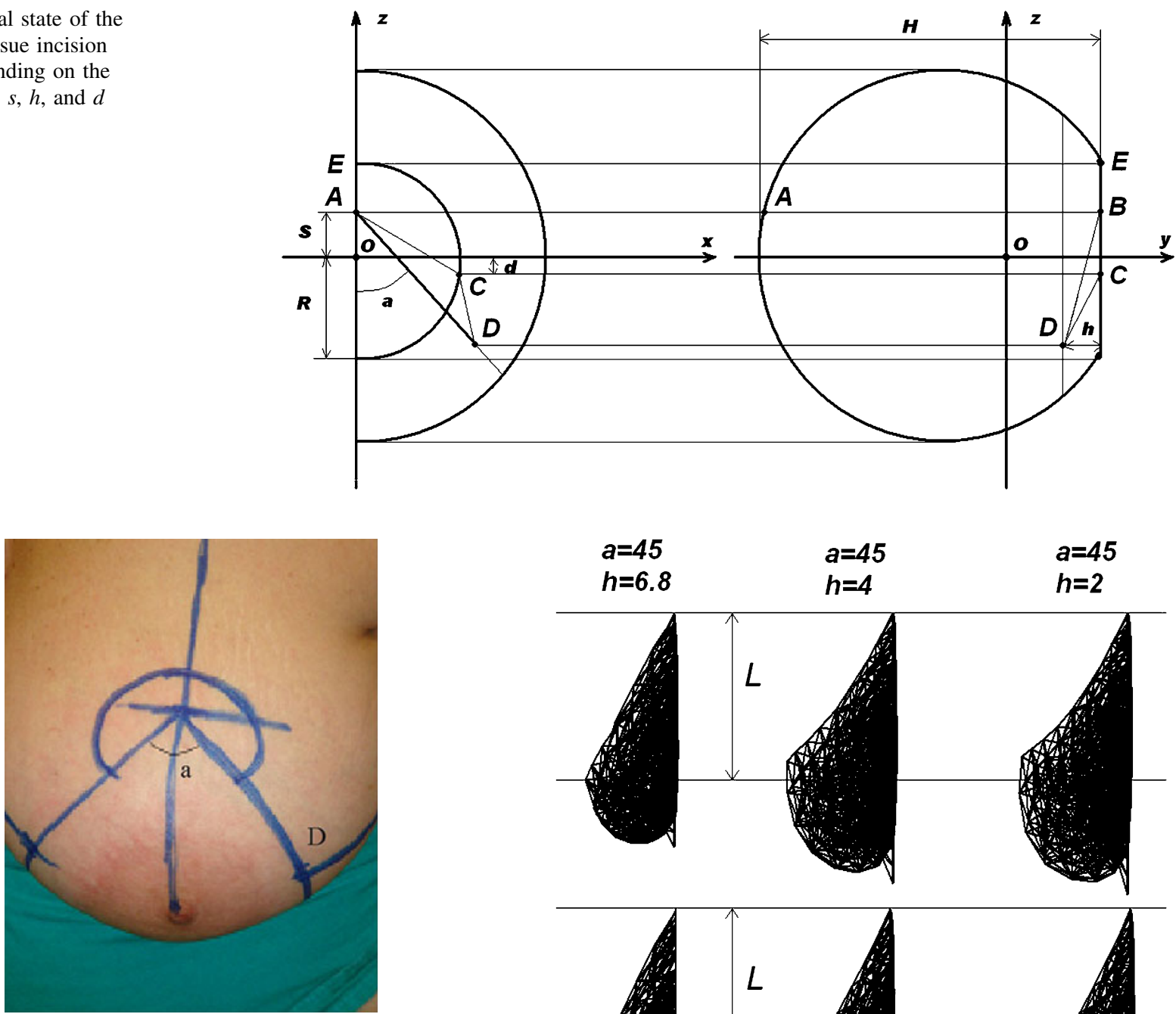

Fig. 9 Angle $a$ and the point $D$

\section{Discussion}

The computer simulation based on biomechanical models allows one to understand many phenomena and to find a solution for the many problems that arise when errors are admitted during the planning for surgery. We did not discuss any special breast reduction technique, but we did study the possibility of modeling any plastic surgery involving a large amount of soft tissue using a computer. The chosen surgery is used as an example.

\section{Nipple Position}

From Fig. 10 we can see how the position of the nipple depends on the choice of parameters $a$ and $h$ defined by the preoperative markings. The height of the nipple on the preoperative breast is the same in all six cases; however, the resulting positions of the nipple when the patient is in the standing position are rather different. From our

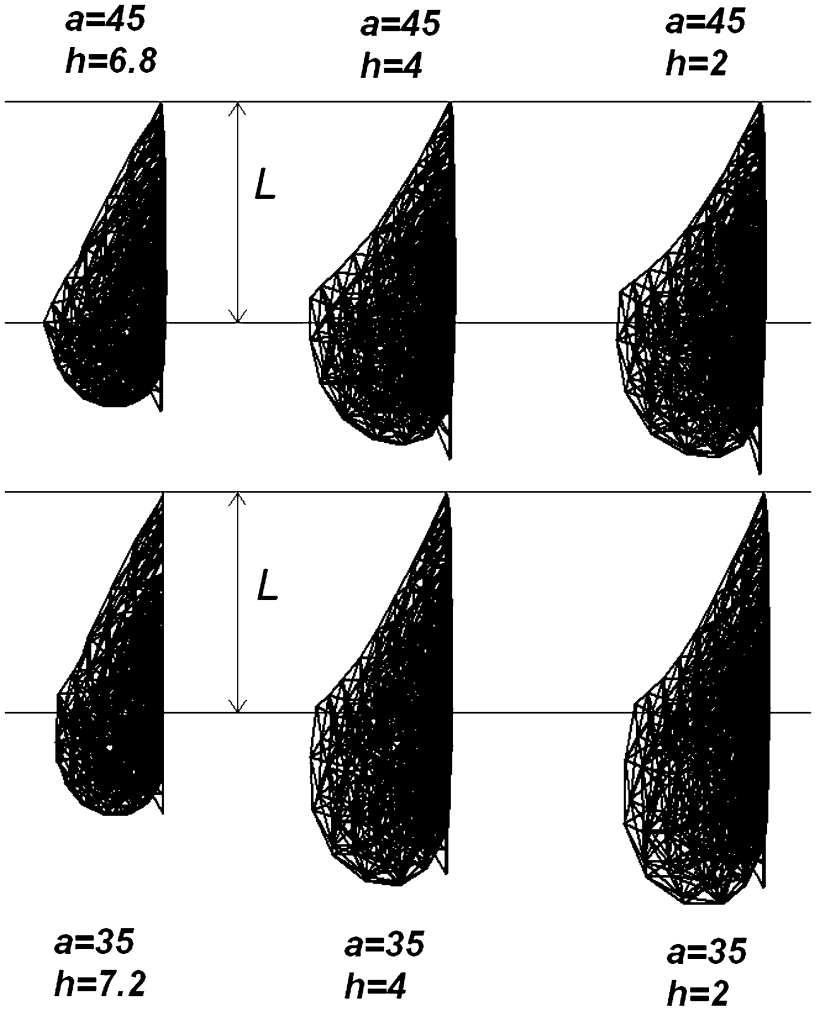

Fig. 10 Results of surgery by varying the parameters $a$ and $h$

simulations it becomes clear that the error in the choice of parameter $h$ causes a high final position of the nipple. This frequently occurs when inferior pedicle reduction mammaplasty is performed [10]. To avoid this error it is necessary to remove much tissue from the lower pole of the breast in the case of a superior pedicle. In the case of an inferior pedicle, it is recommended that the new nipple position be lower than in the case of a superior pedicle. The mark point of the new nipple position is usually the result of the projection of an inframammary fold point onto the 


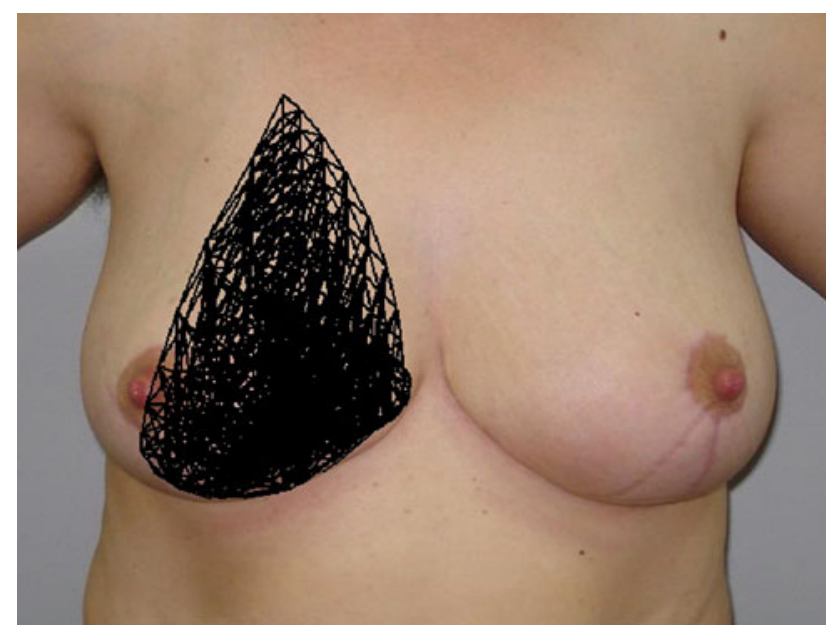

Fig. 11 Comparison of the computer simulation with the final breast form after surgery

midclavicular line. Anthropomorphic studies show that the ideal distance between the nipple and the sternal notch is $21-21.5 \mathrm{~cm}$, the distance between the nipple and the base is $6 \mathrm{~cm}$ (e.g., see [14]).

On the other hand, we see that an insufficient angle $a$ causes excessive tissue in the lower pole of the breast causing it to look "flat."

\section{The Role of the Chassaignac Space}

As we mentioned in the Introduction, the breast has a complex structure involving several tissues. After a series of computational experiments, we came to the conclusion that no realistic breast model is possible without the Chassaignac space, which plays a special role in breast mobility and is responsible for the connection between the breast and the chest. The mobility of the breast base is an experimental fact. For example, we drew the breast's base on the patient's skin. Then we measured the length of this line when the patient was standing and when the patient was supine. The difference between the two measurements was about 20-30 \%. However, the breast cannot arbitrarily

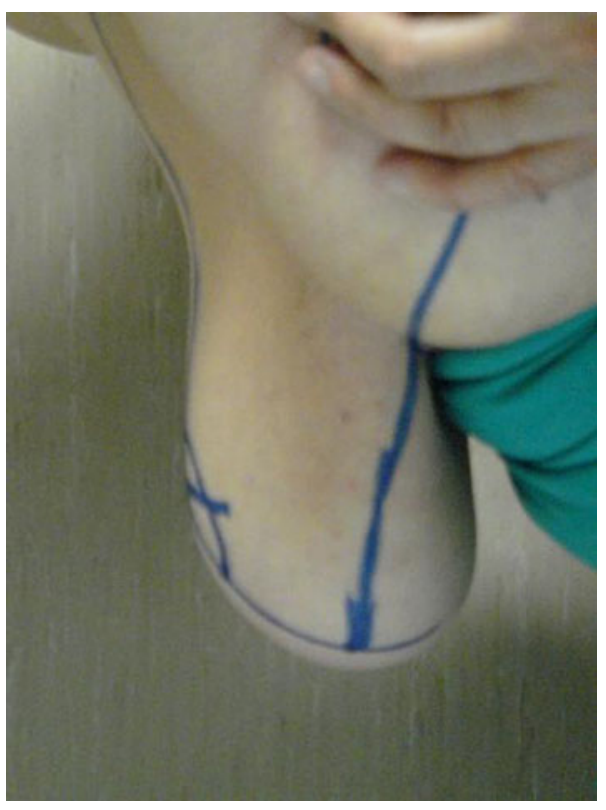

Fig. 12 The concave curve of a breast

move along the body because it is fixed in its lower part, known as the inframammary fold, and by the suspensory ligament in the upper pole. All these tissues should be included in a realistic breast model. We model the Chassaignac space as a mass-spring system. The influence of the Chassaignac space can be seen from the following computational experiment. For the patient in Fig. 12, the angle between the chest and the vertical is about $40^{\circ}$. We can see that the breast has a concave curve in its upper part. This concavity exists because of the Chassaignac space. Indeed, if we increase the stiffness of the springs involved in the modeling of the Chassaignac space, we will get a breast geometry closer to that corresponding to a breast with a fixed base. The breast geometry as a function of spring stiffness that is involved in the Chassaignac space model can be seen in Fig. 13. The stiffness of the springs gradually increases. Figure 13a corresponds to a low stiffness and Fig. 13d corresponds to an almost fixed base. The concavity is seen in the first case and disappears in the
Fig. 13 The breast geometries corresponding to increasing stiffness of the springs involved in the Chassaignac space model

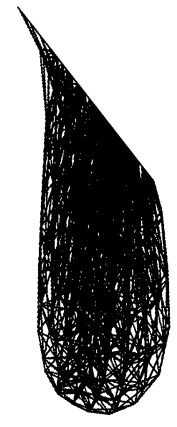

(a)

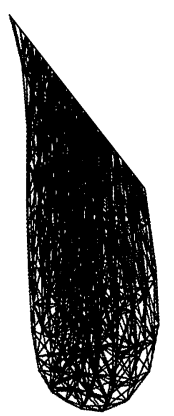

(b)

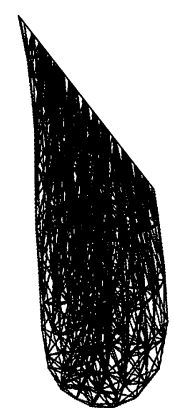

(c)

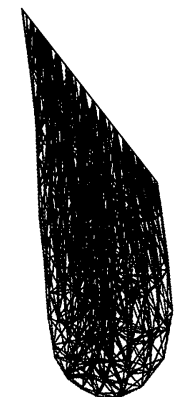

(d) 
second one. This shows the importance of the Chassaignac space in breast geometry modeling.

\section{Conclusions}

Breast reduction is a complex surgical area and an inexperienced surgeon faces serious difficulties in estimating and analyzing the surgical results. The application of computer models based on biomechanical engineering allows simulation of breast reduction with reliable end results. This article presents a method for modeling the techniques of breast reduction. The developed software allows one to estimate the form of the breast after surgery knowing its form before and taking into consideration the parameters of the incision used by the surgeon. We present an example showing how varying the incision parameters can influence the final breast geometry. The images show that the developed methodology can be used for qualitative and quantitative studies of breast reduction surgery with satisfactory results.

For now we have modeled the breast as if the skin is "glued" to the interior body of the breast. In future work we plan to consider more involved breast models that have more realistic geometry and also include the suspensory and Cooper's ligaments.

Our final goal is to offer freeware with a user-friendly interface that can be used in a typical consulting room or a classroom without any special equipment. From the user's point of view, breast modeling will mean the entry of a set of simple parameters to characterize the initial shape of the breast and a set of incision marking parameters. The software will perform the simulation without any user interaction and will propose several visualization options to plot the final results such as the postoperative shape of the breast.

Acknowledgments The authors are grateful to the anonymous reviewers for their comments and valuable suggestions.

Conflict of interest The authors have no conflicts of interest to disclose.

\section{References}

1. Akamatsu M (1993) Identification of Lamé coefficients from tangential boundary observations. J Fac Sci Univ Tokyo Sect IA Math 6:623-634

2. Ayache N, Delingette H (eds) (2003) Surgery simulation and soft tissue modeling, lecture notes in computing science. Springer, Berlin

3. Azar FS, Metaxas DN, Schnall MD (2002) Methods for modeling and predicting mechanical deformations of the breast under external perturbations. Med Image Anal 6:1-27
4. Balaniuk R, Costa I, Melo J (2006) Cosmetic breast surgery simulation. Annals of the VIIIth Symposium on Virtual Reality. Belem, CESUPA

5. Bono JEP (2008) Ligamental mammaplasty. Rev Bras Cir Plást 23(3):192-199

6. Cardoso A, Smirnov G, Costa H, Sá V (2009) Métodos variacionais de engenharia biomecânica em cirurgia plástica de tecidos moles. In: 5th Engineering Conference, Engenharia' 2009 Inovação e Desenvolvimento, Covilhã, 25 a 27 de Novembro, Portugal

7. Delingette H (1998) Towards realistic soft tissue modeling in medical simulation. Proceedings of the IEEE special issues on surgery simulation, pp 512-523

8. Fehrenbach J, Masmoudi M, Souchon R, Trompette P (2006) Relative Young's modulus identification using elastography. Rev Eur Mécan Numér 15:167-174

9. Fung YC (1993) Biomechanics-mechanical properties of living tissues, 2nd edn. Springer, New York

10. Gheita A (2009) Mammaplasty: the "super flap" or the superior pedicle extra long flap for massive breasts with marked ptosis or gigantomasta. Aesthet Plast Surg 33:461-470

11. Gurtin ME (1981) An introduction to continuum mechanics. Academy Press, New York

12. Hall-Findlay EJ (1999) A simplified vertical reduction mammaplasty: shortening the learning curve. Plast Reconstr Surg 104:748-759

13. Hammond DC (1999) Short scar periareolar inferior pedicle reduction (SPAIR) mammaplasty. Plast Reconstr Surg 103(3): 890-901

14. Liu YJ, Thomson JG (2011) Ideal anthropomorphic values of the female breast: correlation of pluralistic aesthetic evaluations with objective measurements. Ann Plast Surg 67:7-11

15. McKissock PK (1979) Reduction mammoplasty. Ann Plast Surg 2:321-331

16. Mollemans W, Schutyser F, Van Cleynenbreugel J, Suetens P (2003) Tetrahedral mass spring model for fast soft tissue deformation. In: Ayache N, Delingette H (eds) Surgery simulation and soft tissue modeling. Lecture Notes in Computer Science, 2673. Springer, Berlin, pp 371-379

17. Perez del Palomar A, Calvo B, Herrero J, López J, Doblaré M (2008) A finite element model to accurately predict real deformations of the breast. Med Eng Phys 30:1089-1097

18. Rajagopal V, Nielsen PMF, Nash MP (2004) Development of a three-dimensional finite element model of breast mechanics. 26th Annual international conference of the IEEE engineering in medicine and biology society: conference proceedings: linkages for innovation in biomedicine, vol 7, San Francisco, 1-5 Sept, pp 5080-5083

19. Retel V, Vescovo P, Jacquet E, Trivaudey F, Varchon D, Burtheret A (2001) Nonlineal model of skin mechanical behaviour analysis with finite element method. Skin Res Technol 7:152-158

20. Robbins TH (1977) A reduction mammoplasty with the areolanipple based on an inferior dermal pedicle. Plast Reconstr Surg 59:64-67

21. Smirnov G, Sá V (2007) Simulação numérica da cirurgia plástica da mama. In: Alves J, Delgado R, Lyra PRM, Oliver JL, Rodrigues-Ferran A, Sá JC, Santos AD (eds) CMNE/CILAMCE 2007, APMTAC/SEMNI, Porto

22. Smirnov G, Sá V (2009) Métodos de determinação dos parâmetros que influenciam os resultados da cirurgia plástica de redução mamária. In: Huerta A, Figueiredo IN, Menezes L, Oñate E, Rodrigues-Ferran A, Tadeu AJB (eds) Congreso de Métodos Numéricos en Ingeniería 2009, SEMNI/APMTAC, Barcelona

23. Spear SL, Howard MA (2003) Evolution of the vertical reduction mammaplasty. Plast Reconstr Surg 112:855-868 
24. Whiteley JP, Gavaghan DJ (2007) Non-linear modeling of breast tissue. Math Med Biol 24:327-345

25. Wise RJ (1956) A preliminary report on a method of planning the mammaplasty. Plast Reconstr Surg 17:367-375
26. Zienkiewicz OC, Taylor RL (2005) The finite element method for solid and structural mechanics, 6th edn. Elsevier, Amsterdam

27. Zienkiewicz OC, Taylor RL, Zhu JZ (2005) The finite element method: its basis and fundamentals, 6th edn. Elsevier, Amsterdam 